\title{
Public perceptions, individual characteristics, and preventive behaviors for COVID-19 in six countries: a cross- sectional study
}

Ryosuke Fujii ${ }^{* \dagger}$ (D) Kensuke Suzuki ${ }^{2+}$ and Junichiro Niimi ${ }^{3}$

\begin{abstract}
Background: Public perceptions and personal characteristics are heterogeneous between countries and subgroups, which may have different impacts on health-protective behaviors during the coronavirus disease 2019 (COVID-19) pandemic. To assess whether self-reported perceptions of COVID-19 and personal characteristics are associated with protective behaviors among general adults and to compare patterns in six different countries.

Methods: This cross-sectional study uses the secondary data collected through an online survey between 15 and 23 April 2020 across six countries (China, Italy, Japan, Korea, the UK, and the USA). A total of 5945 adults aged 18 years or older were eligible for our analysis. A logistic regression model was used to estimate odds ratios (OR) and $95 \%$ confidence intervals $(95 \% \mathrm{Cl})$ of three recommended behaviors (wearing a mask, handwashing, and avoiding social gatherings).

Results: In most countries except for China, the participants who perceived wearing a mask as being extremely effective to curtail the pandemic were more likely to wear a mask (OR, 95\%Cl: Italy: 4.14, 2.08-8.02; Japan: 3.59, 1.75-7.30; Korea: 7.89, 1.91-31.63: UK: 9.23, 5.14-17.31; USA: 4.81, 2.61-8.92). Those who perceived that handwashing was extremely effective had higher ORs of this preventive behavior (OR, 95\%Cl: Italy: 16.39, 3.56-70.18; Japan: 12.24, 4.03-37.35; Korea: 12.41, 2.02-76.39; UK: 18.04, 2.60-152.78; USA: 10.56, 2.21-44.32). The participants who perceived avoiding social gathering as being extremely effective to curtail the pandemic were more likely to take this type of preventive behavior (OR, 95\%Cl: China: 3.79, 1.28-10.23; Korea: 6.18, 1.77-20.60; UK: 4.45, 1.63-11.63; USA: 4.34, 1.849.95). The associations between personal characteristics, living environment, psychological status, and preventive behaviors varied across different countries. Individuals who changed their behavior because of recommendations from doctors/public health officials were more likely to take preventive behaviors in many countries.

(Continued on next page)
\end{abstract}

\footnotetext{
* Correspondence: rfujii@fujita-hu.ac.jp

${ }^{\dagger}$ Ryosuke Fujii and Kensuke Suzuki contributed equally to this work.

'Department of Preventive Medical Sciences, Fujita Health University School

of Medical Sciences, 1-98 Dengakugakubo, Kutsukake-cho, Toyoake 470-1192,

Japan

Full list of author information is available at the end of the article
}

(c) The Author(s). 2021 Open Access This article is licensed under a Creative Commons Attribution 4.0 International License, which permits use, sharing, adaptation, distribution and reproduction in any medium or format, as long as you give appropriate credit to the original author(s) and the source, provide a link to the Creative Commons licence, and indicate if changes were made. The images or other third party material in this article are included in the article's Creative Commons licence, unless indicated otherwise in a credit line to the material. If material is not included in the article's Creative Commons licence and your intended use is not permitted by statutory regulation or exceeds the permitted use, you will need to obtain permission directly from the copyright holder. To view a copy of this licence, visit http://creativecommons.org/licenses/by/4.0/. The Creative Commons Public Domain Dedication waiver (http://creativecommons.org/publicdomain/zero/1.0/) applies to the data made available in this article, unless otherwise stated in a credit line to the data. 
(Continued from previous page)

Conclusions: These findings suggest that higher perceived effectiveness may be a common factor to encourage preventive behaviors in response to the COVID-19 pandemic. These results may provide a better understanding of the homogeneity and heterogeneity of factors related to preventive behaviors and improve public health policies in various countries and groups.

Keywords: COVID-19, Public perception, Preventive behavior, Wearing a mask, Handwashing, Avoiding social gatherings

\section{Background}

In December 2019, an outbreak of coronavirus disease 2019 (COVID-19) was identified in Wuhan, China. From mid-January to February 2020, the virus spread to Asian countries, and over the next few months, around the world [1]. As of 14 January 2021, a total of 92,888,380 cases and 1989,349 deaths due to the COVID-19 had been reported around the world [2]. The spread of the pandemic over time has varied substantially across not only countries but also regions within countries. Many plausible reasons underlie this geographic variation, such as the prevalence of preexisting conditions $[3,4]$, administrative orders, and recommendations for slowing the spread $[5,6]$.

Given the lack of effective pharmaceutical interventions against COVID-19, preventive measures at the individual level have been crucial to reduce the risk of infection. In fact, some recent papers have suggested that wearing a mask and handwashing are effective to prevent the spread of severe acute respiratory syndromecoronavirus-2 (SARS-CoV-2) [7-9]. Many previous interdisciplinary studies have attempted to comprehend the determinants of individual preventive behaviors using established conceptual frameworks, e.g., the Health Belief Model (HBM). According to these studies, engaging in preventive behaviors may be triggered by a complex combination of constructs, such as barriers, optimistic biases, social contexts, science communication, and personal perceptions/beliefs [10-14]. In addition, diverse factors underlying individual perceptions, such as demographic, psychological, and socioeconomic characteristics, need to be considered [15].

Since the outbreak of COVID-19, a growing number of studies have attempted to predict the spread of the disease using mathematical modeling [16-18]. In those studies, researchers set parameters that govern individual behavior and the transmission of the disease among populations and impact the simulation results substantially. Therefore, understanding the interactions between individual demographic and socioeconomic characteristics, cultural and social contexts, perceptions about the disease, and health preventive behaviors is a fundamental step to develop advanced modeling and provide critical insights into how public health experts should best respond to the pandemic.
Given that factors related to preventive behaviors are country-dependent, in this cross-sectional study, we aimed to describe international differences in personal characteristics, cultural backgrounds, and perceptions about the pandemic, and to examine the associations between these factors and health preventive behaviors in six countries.

\section{Methods \\ Cross-sectional online survey}

In order to analyze the relationship between public perceptions, individual characteristics, and preventive behaviors for COVID-19 in six countries, this crosssectional study was a secondary analysis using information obtained through an online survey conducted across six different countries (China, Italy, Japan, Korea, the UK, and the USA) from 15 to 23 April 2020 [19]. The online survey was performed with the support of two market research companies (Lucid Holdings, LLC, New Orleans, LA, and dataSpring, Inc., Tokyo, Japan; the data are publicly available at https://osf.io/aubkc/). The interview form was developed for this study and is available as Additional file 1 in the English version. Before participating in the online survey, informed consent was obtained from all participants after specifying that anonymized individual-level data would be made public. Participation was remunerated according to general compensation schemes defined by each company. The median response time to complete the questionnaire was about $14 \mathrm{~min}$. To ensure the reliability of the survey, those who responded at less than $50 \%$ of the median response time were excluded beforehand. The original study protocol was approved by the ethics board at the University of Exeter (eUEBS003014 v2.0), covering surveys in all countries in the midst of a time-sensitive crisis. In addition, the protocol was officially reviewed by the ethics review board of Fujita Health University (HM20-182).

\section{Study population}

A total of 6089 adults aged 18 years or older were eligible for this survey. As described in [19], the participants are confirmed to be nationally representative in terms of age structure, sex, and household income in 
each country. We excluded 144 individuals from the analysis because of missing values on the questionnaire, resulting in a data set consisting of 5945 individuals (2900 men and 3045 women).

\section{Public perceptions}

Perceived susceptibility was assessed according to whether participants believed that they are or had been infected with SARS-CoV-2. Perceived severity was assessed according to whether participants believed that they would develop a serious illness requiring hospitalization because of the virus. These question items were answered in terms of percentage points $(0-$ $100 \%)$. The perceived effectiveness of each preventive behavior was assessed according to whether participants believed that a specific action would help slow the spread of COVID-19 based on the following five options: (1) not effective at all, (2) slightly effective, (3) moderately effective, (4) very effective, or (5) extremely effective. The original questions and items in the survey regarding public perceptions are summarized in the upper part of Table 1.

\section{Preventive behaviors}

Participants were asked questions regarding the frequency of various daily behaviors. The questions about each behavior were prefaced by the statement, "Please tell us how often you have engaged in each of the following behaviors." The participants responded to the question items about these behaviors on a five-point scale (never, rarely, sometimes, very often, or always). We selected two behaviors that had been recommended by government offices and national official institutes: wearing a mask and either handwashing with water and soap or using hand sanitizer. The participants who answered "never" or "rarely" were defined as those who were not taking preventive behaviors, whereas those who answered "sometimes," "very often," or "always" were defined as those who were taking preventive behaviors. By contrast, we selected one unfavorable behavior-participation in a social gathering with more than 20 peoplefor which the participants who answered "never" or "rarely" were defined as those who were taking preventive behaviors, whereas those who answered "sometimes," "very often," or "always" were defined as those who were not taking preventive behaviors. Participants were also asked questions regarding the different reasons why they changed their daily behaviors. In this study, we selected the variables of recommendations from family/friends, doctors/public health officials, or politicians, and conformity. Since the questionnaire did not specify the changed behavior, the responses to those questions should be interpreted as representing how likely an individual's behavior was affected by conformity or

Table 1 List of variables for public perception and preventive behaviors in this study (compared with the questions and values in) and the original questionnaire

\begin{tabular}{|c|c|c|c|}
\hline Variable name in this study & Original survey question & Values in the original survey & Values in this study \\
\hline Perceived susceptibility & $\begin{array}{l}\text { What do you think is the probability } \\
\text { that you are or have been infected } \\
\text { with COVID-19? }\end{array}$ & $\begin{array}{l}\text { Probability in percentage points } \\
\% \text { (integers } 0-100 \text { ) }\end{array}$ & Same as questionnaire \\
\hline \multirow[t]{2}{*}{ Perceived severity } & $\begin{array}{l}\text { What do you think is the probability } \\
\text { that an infected person develops a } \\
\text { serious illness that requires } \\
\text { hospitalization? }\end{array}$ & \multirow[t]{2}{*}{$\begin{array}{l}\text { Probability in percentage points } \\
\% \text { (integers 0-100) }\end{array}$} & \multirow[t]{2}{*}{ Same as questionnaire } \\
\hline & $\begin{array}{l}\text { How effective do you believe each } \\
\text { of these measures is in reducing the } \\
\text { spread of the epidemic? }\end{array}$ & & \\
\hline $\begin{array}{l}\text { Perceived effectiveness of } \\
\text { wearing a mask }\end{array}$ & $\begin{array}{l}\text { Requiring masks to be worn outside } \\
\text { by everyone }\end{array}$ & \multirow{4}{*}{$\begin{array}{l}1=\text { Not effective at all, } 2 \text { = Slightly } \\
\text { effective, } 3 \text { = Moderately effective, } \\
4=\text { Very effective, } 5 \text { = Extremely } \\
\text { effective }\end{array}$} & \multirow[t]{4}{*}{ Same as questionnaire } \\
\hline $\begin{array}{l}\text { Perceived effectiveness of } \\
\text { handwashing }\end{array}$ & Hand washing & & \\
\hline \multirow{2}{*}{$\begin{array}{l}\text { Perceived effectiveness of } \\
\text { avoiding social gatherings }\end{array}$} & Forbidding mass gatherings & & \\
\hline & $\begin{array}{l}\text { For each of the following behaviors, } \\
\text { please tell us how often you engaged } \\
\text { in them NOW }\end{array}$ & & \\
\hline Wearing a mask & Wear a mask & \multirow{3}{*}{$\begin{array}{l}1=\text { Never }, 2=\text { Rarely, } 3=\text { Sometimes, } \\
4=\text { Very often, } 5=\text { Always }\end{array}$} & \multirow{3}{*}{$\begin{array}{l}1 \text { or } 2 \text { : NOT taking } \\
\text { preventive behaviors } \\
3,4 \text {, or } 5 \text { : taking } \\
\text { preventive behaviors }\end{array}$} \\
\hline Handwashing & $\begin{array}{l}\text { Wash your hands with water and soap, } \\
\text { or use hand sanitizer }\end{array}$ & & \\
\hline Avoiding social gatherings & $\begin{array}{l}\text { Participate in a social gathering } \\
\text { with more than } 20 \text { people }\end{array}$ & & \\
\hline
\end{tabular}

${ }^{a}$ The detail information of this questionnaire is available in https://osf.io/3pxkr/ 
recommendations from others during the pandemic. The questions and items regarding health behaviors are summarized in the bottom part of Table 1 .

\section{Statistical analysis}

We calculated means and standard deviations for continuous variables and numbers and percentages for categorical variables. We performed country-stratified logistic regression analyses to estimate the multivariable association between public perceptions, personal characteristics, and the three preventive behaviors. We included the following variables in our analyses: sex, age (65 years or older), residential area, living arrangements, income level (five groups), perceived probability, perceived severity, perceived effectiveness, and feeling anxiety, and the variable of region as a covariate. All statistical analyses were performed using $\mathrm{R}$ version 4.0.0 ( $\mathrm{R}$ Foundation for Statistical Computing, Vienna, Austria). The codes used for the statistical analysis are uploaded to GitHub (https://github.com/fujichaaan/ covid19_opendata).

\section{Results}

Table 2 shows the respondents' characteristics by country. The proportions of respondents who answered that he/she wore a mask (at the time of the survey) were above $85 \%$ in three Asian countries and Italy, compared with $73.3 \%$ and $27.6 \%$ in the UK and the USA, respectively. Over $90 \%$ of respondents washed their hands/used hand sanitizer in all countries except for Japan. Finally, the proportions of respondents who avoided social gatherings ranged from $81.5 \%$ in the USA to $92.6 \%$ in Japan.

Table 3 shows the associations between public perceptions, personal characteristics, and wearing a mask by country. Lower odds ratios (ORs) were observed with a $10 \%$ increment of perceived susceptibility in China (OR, 0.83; 95\% confidence interval [CI], 0.74-0.94) and Korea (OR, 0.86; 95\%CI, 0.75-1.00), and the UK (OR, 1.08; 95 $\% \mathrm{Cl}, 1.01-1.14)$. High perceived severity was associated with wearing a mask in the USA $(\mathrm{OR}, 1.07$; 95\% CI, 1.02-1.14), whereas a negative association was observed in China (OR, 0.91; $95 \% \mathrm{Cl}, 0.83-0.99$ ). Except for China, those who perceived that wearing a mask was extremely effective had higher ORs in Italy (OR, 4.14; 95\%CI, 2.08-8.02), Japan (OR, 3.59; 95\%CI, 1.75-7.30), Korea (OR, 7.89; 95\%CI, 1.91-31.63), the UK (OR, 9.23; 95\% CI, 5.14-17.31), and the USA (OR, 4.81; 95\%CI, 2.61-8.92). Older people were less likely to wear a mask in the UK (OR, 0.29; 95\%CI, 0.15-0.51). Male participants were less likely to wear a mask only in Japan (OR, 0.30 ; 95\%CI, 0.19-0.46). Those who lived in urban areas had high ORs in Japan (OR, 1.70; 95\%CI, 1.03-2.90), the UK (OR, 1.52; 95\%CI, 1.09-2.11), and the USA (OR, $1.59 ; 95 \% \mathrm{CI}, 1.15-2.20)$. The participants in the fifth income quintile were more likely to wear a mask in Korea
(OR, 9.19; 95\%CI, 2.50-59.50), and the USA (OR, 3.24; $95 \% \mathrm{CI}, 1.93-5.53)$. Feeling anxiety was positively associated with wearing a mask in Japan (OR, 2.11; 95\%CI, $1.37-3.31)$, whereas those who were living alone were less likely to wear a mask (OR, 0.43; 95\%CI, 0.28-0.68).

Table 4 shows the associations between public perceptions, personal characteristics, and handwashing/using hand sanitizers by country. Inverse associations were observed between hand sanitizing and perceived susceptibility in China (OR, 0.81; 95\%CI, 0.71-0.91) and Korea (OR, $0.77 ; 95 \% \mathrm{CI}, 0.66-0.90)$. Those who had higher perceived effectiveness were more likely to wash their hands in Italy (OR, 16.39; 95\%CI, 3.56-70.18), Japan (OR, 12.24; 95\%CI, 4.03-37.35), Korea (OR, 12.41; 95\%CI, 2.02-76.39), the UK (OR, 18.04; 95\%CI, 2.60-152.78), and the USA (OR, 10.56; 95\%CI, 2.21-44.32). In line with wearing a mask, older people were more likely to wash their hands in Japan (OR, 2.47; 95\%CI, 1.27-5.21). Male respondents were less likely to wash their hands in Japan (OR, 0.32; 95\%CI, 0.19-0.53) and China (OR, 0.56; 95\%CI, 0.31-0.98). Those who lived alone were less likely to wash their hands in Japan (OR, 0.50; 95\%CI, 0.31-0.82). Feeling anxiety was positively associated with washing hands in China (OR, 2.05; 95\% CI, 1.15-3.80) and Japan (OR, 1.74; 95\%CI, $1.09-2.83)$. In Korea only, the participants in the fifth income quintile were more likely to wash hands (OR, 3.50; 95\%CI, 1.27-11.37)

Table 5 shows the associations between public perceptions, personal characteristics, and avoiding social gatherings in the six different countries. Perceived susceptibility was inversely associated with avoiding social gatherings in China (OR, 0.74; 95\%CI, 0.66-0.83), Japan (OR, 0.85; 95\%CI, 0.77-0.95), Korea (OR, 0.81; 95\%CI, $0.73-0.91$ ), and the USA (OR, 0.83; 95\%CI, 0.77-0.90). Participants with high perceived severity were more likely to avoid social gatherings in China (OR, 1.27; 95\% CI, 1.16-1.41) and the USA (OR, 1.11; 95\%CI, 1.031.19). Those who had higher perceived effectiveness were more likely to avoid social gatherings in China (OR, 3.79; 95\%CI, 1.28-10.23), Korea (OR, 6.18; 95\%CI, 1.77-20.60), the UK (OR, 4.45; 95\%CI, 1.63-11.63), and the USA (OR, 4.34; 95\%CI, 1.84-9.95), but neither in Italy nor in Japan. Older people tended to avoid social gatherings in China (OR, 2.78; 95\% CI, 1.29-6.55). Male participants were less likely to avoid social gatherings in Japan (OR, 0.33; 95\%CI, 0.19-0.58) and the USA (OR, 0.54; 95\%CI, 0.36-0.82). Although those who lived in urban areas were more likely to avoid social gatherings in Korea (OR, 2.24; 95\%CI, 1.453.46 ), an inverse association was found in Italy (OR, 0.43 ; 95\% CI, 0.24-0.75) and the UK (OR, 0.49; 95\% CI, 0.31-0.78). Participants with higher incomes were less likely to avoid social gatherings in the USA (OR, 0.49; 95\%CI, 0.24-0.97). 
Table 2 Public perception and personal characteristics of participants by six countries

\begin{tabular}{|c|c|c|c|c|c|c|}
\hline & China $(n=994)$ & Italy $(n=1020)$ & Japan $(n=981)$ & Korea $(n=918)$ & UK $(n=994)$ & US $(n=1038$ \\
\hline Over 65 years old $(n)$ & $117(11.8 \%)$ & $180(17.6 \%)$ & $196(20.0 \%)$ & $133(14.5 \%)$ & $157(15.8 \%)$ & $239(23.0 \%)$ \\
\hline Male (n) & $487(49.0 \%)$ & $522(51.2 \%)$ & $474(48.3 \%)$ & $474(51.6 \%)$ & 489 (49.2\%) & $454(43.7 \%)$ \\
\hline Living urban (n) & $723(72.7 \%)$ & $607(59.5 \%)$ & $271(27.6 \%)$ & $554(60.3 \%)$ & $339(34.1 \%)$ & $428(41.2 \%)$ \\
\hline Living alone $(n)$ & $418(42.1 \%)$ & $168(16.5 \%)$ & $218(22.2 \%)$ & $155(16.9 \%)$ & $233(23.4 \%)$ & $253(24.4 \%)$ \\
\hline \multicolumn{7}{|l|}{ Income group (n) } \\
\hline First quintile & $201(20.2 \%)$ & $170(16.7 \%)$ & $206(21.0 \%)$ & $198(21.6 \%)$ & $180(18.1 \%)$ & $181(17.4 \%)$ \\
\hline Second quintile & 199 (20.0\%) & $179(17.5 \%)$ & $209(21.3 \%)$ & $163(17.8 \%)$ & $179(18.0 \%)$ & 195 (18.8\%) \\
\hline Third quintile & 198 (19.9\%) & $244(23.9 \%)$ & $214(21.8 \%)$ & 199 (21.7\%) & $196(19.7 \%)$ & $218(21.0 \%)$ \\
\hline Fourth quintile & $198(19.9 \%)$ & $262(25.7 \%)$ & $187(19.1 \%)$ & $200(21.8 \%)$ & $222(22.3 \%)$ & 245 (23.6\%) \\
\hline Fifth quintile & $198(19.9 \%)$ & $165(16.2 \%)$ & $165(16.8 \%)$ & $158(17.2 \%)$ & $217(21.8 \%)$ & 199 (19.2\%) \\
\hline Feeling anxiety $(n)$ & $450(45.3 \%)$ & $468(45.9 \%)$ & $472(48.1 \%)$ & $437(47.6 \%)$ & $431(43.4 \%)$ & 447 (43.1\%) \\
\hline Use public transportation ( $n$ ) & $269(27.1 \%)$ & $96(9.4 \%)$ & $264(26.9 \%)$ & $332(36.2 \%)$ & $179(18.0 \%)$ & $147(14.2 \%)$ \\
\hline Perceived susceptibility (\%) ${ }^{a}$ & $10.0(16.6)$ & $14.0(22.7)$ & $19.8(22.6)$ & $10.7(17.5)$ & $22.4(26.2)$ & $21.7(28.4)$ \\
\hline Perceived severity $(\%)^{a}$ & $35.3(30.8)$ & $31.3(26.3)$ & $27.8(23.3)$ & $28.5(28.0)$ & $31.9(25.2)$ & $40.3(29.6)$ \\
\hline \multicolumn{7}{|c|}{ Perceived effectiveness for wearing a mask ( $n$ ) } \\
\hline Not effective at all & $22(2.2 \%)$ & $61(6.0 \%)$ & $69(7.0 \%)$ & $14(1.5 \%)$ & $197(19.8 \%)$ & $58(5.6 \%)$ \\
\hline Slightly effective & $31(3.1 \%)$ & $54(5.3 \%)$ & $148(15.1 \%)$ & $28(3.1 \%)$ & $207(20.8 \%)$ & $107(10.3 \%)$ \\
\hline Moderately effective & $105(10.6 \%)$ & $150(14.7 \%)$ & $248(25.3 \%)$ & $127(13.8 \%)$ & $272(27.4 \%)$ & $204(19.7 \%)$ \\
\hline Very effective & $263(26.5 \%)$ & $272(26.7 \%)$ & $213(21.7 \%)$ & $394(42.9 \%)$ & $164(16.5 \%)$ & $285(27.5 \%)$ \\
\hline Extremely effective & $573(57.6 \%)$ & $483(47.4 \%)$ & $303(30.9 \%)$ & $355(38.7 \%)$ & $154(15.5 \%)$ & $384(37.0 \%)$ \\
\hline \multicolumn{7}{|c|}{ Perceived effectiveness for washing hands ( $n$ ) } \\
\hline Not effective at all & $19(1.9 \%)$ & $18(1.8 \%)$ & $19(1.9 \%)$ & $9(1.0 \%)$ & $21(2.1 \%)$ & $15(1.4 \%)$ \\
\hline Slightly effective & $32(3.2 \%)$ & $46(4.5 \%)$ & $92(9.4 \%)$ & $30(3.3 \%)$ & $60(6.0 \%)$ & $47(4.5 \%)$ \\
\hline Moderately effective & $151(15.2 \%)$ & $153(15.0 \%)$ & $178(18.1 \%)$ & $141(15.4 \%)$ & $202(20.3 \%)$ & $117(11.3 \%)$ \\
\hline Very effective & $444(44.7 \%)$ & $379(37.2 \%)$ & $316(32.2 \%)$ & $479(52.2 \%)$ & $322(32.4 \%)$ & $295(28.4 \%)$ \\
\hline Extremely effective & $348(35.0 \%)$ & $424(41.6 \%)$ & $376(38.3 \%)$ & $259(28.2 \%)$ & $389(39.1 \%)$ & $564(54.3 \%)$ \\
\hline \multicolumn{7}{|c|}{ Perceived effectiveness for avoiding social gathering $(n)$} \\
\hline Not effective at all & $34(3.4 \%)$ & $31(3.0 \%)$ & $59(6.0 \%)$ & $19(2.1 \%)$ & $34(3.4 \%)$ & $37(3.6 \%)$ \\
\hline Slightly effective & $32(3.2 \%)$ & $38(3.7 \%)$ & $69(7.0 \%)$ & $35(3.8 \%)$ & $38(3.8 \%)$ & $64(6.2 \%)$ \\
\hline Moderately effective & $133(13.4 \%)$ & $71(7.0 \%)$ & $163(16.6 \%)$ & $90(9.8 \%)$ & $125(12.6 \%)$ & $118(11.4 \%)$ \\
\hline Very effective & $265(26.7 \%)$ & $233(22.8 \%)$ & $209(21.3 \%)$ & $339(36.9 \%)$ & $256(25.8 \%)$ & $250(24.1 \%)$ \\
\hline Extremely effective & $530(53.3 \%)$ & $647(63.4 \%)$ & $481(49.0 \%)$ & $435(47.4 \%)$ & $541(54.4 \%)$ & $569(54.8 \%)$ \\
\hline \multicolumn{7}{|l|}{ Wearing a mask $(n)$} \\
\hline Never & $24(2.4 \%)$ & $75(7.4 \%)$ & $59(6.0 \%)$ & $27(2.9 \%)$ & $661(66.5 \%)$ & $206(19.8 \%)$ \\
\hline Rarely & $48(4.8 \%)$ & $55(5.4 \%)$ & $63(6.4 \%)$ & $26(2.8 \%)$ & $59(5.9 \%)$ & $71(6.8 \%)$ \\
\hline Sometimes & $68(6.8 \%)$ & $66(6.5 \%)$ & $98(10.0 \%)$ & $41(4.5 \%)$ & $90(9.1 \%)$ & $143(13.8 \%)$ \\
\hline Very often & $209(21.0 \%)$ & $164(16.1 \%)$ & $184(18.8 \%)$ & $155(16.9 \%)$ & $76(7.6 \%)$ & $212(20.4 \%)$ \\
\hline Always & $645(64.9 \%)$ & $660(64.7 \%)$ & $577(58.8 \%)$ & $669(72.9 \%)$ & $108(10.9 \%)$ & $406(39.1 \%)$ \\
\hline \multicolumn{7}{|c|}{ Handwashing/using hand sanitizers ( $n$ ) } \\
\hline Never & $8(0.8 \%)$ & $13(1.3 \%)$ & $47(4.8 \%)$ & $28(3.1 \%)$ & $7(0.7 \%)$ & $16(1.5 \%)$ \\
\hline Rarely & $56(5.6 \%)$ & $15(1.5 \%)$ & $52(5.3 \%)$ & $23(2.5 \%)$ & $19(1.9 \%)$ & $26(2.5 \%)$ \\
\hline Sometimes & $60(6.0 \%)$ & $38(3.7 \%)$ & $75(7.6 \%)$ & $48(5.2 \%)$ & $75(7.5 \%)$ & $65(6.3 \%)$ \\
\hline Very often & $320(32.2 \%)$ & $285(27.9 \%)$ & 199 (20.3\%) & $279(30.4 \%)$ & $303(30.5 \%)$ & $228(22.0 \%)$ \\
\hline Always & $550(55.3 \%)$ & 669 (65.6\%) & $608(62.0 \%)$ & $540(58.8 \%)$ & $590(59.4 \%)$ & 703 (67.7\%) \\
\hline
\end{tabular}


Table 2 Public perception and personal characteristics of participants by six countries (Continued)

\begin{tabular}{lllllll}
\hline & China $(\boldsymbol{n}=\mathbf{9 9 4})$ & Italy $(\boldsymbol{n}=\mathbf{1 0 2 0})$ & Japan $(\boldsymbol{n}=\mathbf{9 8 1})$ & Korea $(\boldsymbol{n = 9 1 8 )}$ & UK $(\boldsymbol{n}=\mathbf{9 9 4})$ & US $(\boldsymbol{n}=\mathbf{1 0 3 8})$ \\
\hline Avoiding social gathering $(n)$ & & & & & & \\
$\quad$ Never & $695(69.9 \%)$ & $864(84.7 \%)$ & $806(82.2 \%)$ & $664(72.3 \%)$ & $742(74.6 \%)$ & $698(67.2 \%)$ \\
Rarely & $167(16.8 \%)$ & $58(5.7 \%)$ & $102(10.4 \%)$ & $116(12.6 \%)$ & $112(11.3 \%)$ & $148(14.3 \%)$ \\
Sometimes & $73(7.3 \%)$ & $42(4.1 \%)$ & $42(4.3 \%)$ & $47(5.1 \%)$ & $73(7.3 \%)$ & $87(8.4 \%)$ \\
Very often & $41(4.1 \%)$ & $27(2.6 \%)$ & $13(1.3 \%)$ & $37(4.0 \%)$ & $34(3.4 \%)$ & $65(6.3 \%)$ \\
Always & $18(1.8 \%)$ & $29(2.8 \%)$ & $18(1.8 \%)$ & $54(5.9 \%)$ & $33(3.3 \%)$ & $40(3.9 \%)$ \\
\hline
\end{tabular}

${ }^{a}$ Values are expressed as mean and standard deviation

Table 6 shows the associations between behavioral changes and the three preventive measures. Those who changed their behaviors because of recommendations from doctors were more likely to wear a mask in China (OR, 2.04; 95\%CI, 1.12-3.78), Italy (OR, 2.02; 95\%CI, 1.34-3.06), Japan (OR, 2.84; 95\%CI, 1.34-7.01), and the USA (OR, 1.82; 95\%CI, 1.33-2.50). The same trend was observed with handwashing/using hand sanitizers in China (OR, 1.70; 95\%CI, 0.92-3.20), Italy (OR, 2.42; 95\%CI, 0.98-6.34), and Korea (OR, 2.85; 95\%CI, 0.9512.42). Regarding avoiding social gatherings, a significant association with recommendations from doctors/public health officials the USA (OR, 2.14; 95\%CI, 1.40-3.28). Statistically significant associations were observed only between behavioral changes triggered by politicians and wearing masks in the USA (OR, 1.63; 95\%CI, 1.15-2.33) and avoiding social gatherings in the UK (OR, 1.70; $95 \% \mathrm{CI}, 1.05-2.79)$. Japan was the only country in which a statistically significant positive association was observed between behavioral changes triggered by conformity (OR, 5.18; 95\%CI, 1.54-32.35) and recommendations by family members to wear a mask (OR, 3.42; 95\%CI, 1.19-14.48). On the other hand, in the UK, wearing a mask were inversely associated with recommendations from doctors (OR, 0.66; 95\% CI, 0.48-0.90) or politicians (OR, 0.56; 95\%CI, 0.41-0.77).

\section{Discussion}

In this study, we examined the association between perceptions, personal characteristics, recommendations from others, and three preventive behaviors in six countries during the COVID-19 pandemic. According to the HBM, a canonical model of behavioral medicine, the three individual perceptions examined here affect the preventive measures to be taken [20]. Among these three perceptions, we found that perceived effectiveness was a common driving factor for engaging in preventive behaviors in all six countries. Regarding the other two perceptions, we found that the effects varied across countries. Associations between other individual demographic and socioeconomic traits and preventive behaviors were also heterogeneous across countries.
In behavioral medicine, it is widely accepted that perceived susceptibility (likelihood of contracting a disease or developing a condition), perceived severity (seriousness of an illness), and perceived effectiveness (effectiveness of a suggested preventive measure) are key components in taking preventive measures and thereby controlling infectious disease outbreaks. During the influenza A virus subtype H1N1 pandemic of 2009, both perceived susceptibility and severity were found to be significant factors in persuading the public to change their preventive behavior [10-12]. Contrary to our theoretical expectations, however, our results showed inverse associations between perceived susceptibility and severity and preventive behaviors in some countries. This could potentially be attributed to reverse causality, i.e., we hypothesized that because of the cross-sectional design, the respondents who took preventive behaviors were more likely to perceive a low likelihood of having been infected. In addition, the design of the questionnaire might have failed to gauge empirically the concept of perceived susceptibility. Therefore, the association observed in this study between preventive behaviors and perceived susceptibility requires careful interpretation. Through the lens of the HBM, perceived effectiveness is a concept similar to the construct of "perceived benefit." Previous studies have reported positive associations between an individual's perceived effectiveness and preventive behaviors, e.g., during the SARS epidemic of 2003, people who perceived the effectiveness of preventive measures in Hong Kong were more likely to wear a mask (OR: 7.15, 95\%CI: 4.25-12.05), wash their hands (OR: 32.00, 95\%CI: 13.88-73.78), and avoid crowded places (OR: 31.56, 95\%CI: 15.61-63.82) [14]. Considering the observed homogeneous effects of perceived effectiveness across preventive behaviors and countries, government recommendations and social communications should enhance their effectiveness to promote better compliance.

Although the results of this study were not uniform across all countries, personal characteristics, including sex, age, and income groups, were associated with all three preventive behaviors. Similar to the previously reported effect of sex on health behaviors, female 
Table 3 Associations between public perceptions, personal characteristics, and wearing a mask

\begin{tabular}{|c|c|c|c|c|c|c|}
\hline & $\begin{array}{l}\text { China } \\
\text { OR }(95 \% \mathrm{Cl})\end{array}$ & $\begin{array}{l}\text { Italy } \\
\text { OR (95\%Cl) }\end{array}$ & $\begin{array}{l}\text { Japan } \\
\text { OR }(95 \% \mathrm{Cl})\end{array}$ & $\begin{array}{l}\text { Korea } \\
\text { OR }(95 \% \mathrm{Cl})\end{array}$ & $\begin{array}{l}\text { UK } \\
\text { OR (95\%Cl) }\end{array}$ & $\begin{array}{l}\text { US } \\
\text { OR }(95 \% \mathrm{Cl})\end{array}$ \\
\hline \multicolumn{7}{|l|}{ Age group } \\
\hline Under 65 years old & 1.00 (Ref.) & 1.00 (Ref.) & 1.00 (Ref.) & 1.00 (Ref.) & 1.00 (Ref.) & 1.00 (Ref.) \\
\hline Over 65 years old & $1.05(0.49-2.45)$ & $0.92(0.54-1.61)$ & $1.41(0.83-2.46)$ & $0.85(0.39-2.07)$ & $0.29(0.15-0.51)^{b}$ & $1.29(0.89-1.88)$ \\
\hline \multicolumn{7}{|l|}{ Sex } \\
\hline Female & 1.00 (Ref.) & 1.00 (Ref.) & 1.00 (Ref.) & 1.00 (Ref.) & 1.00 (Ref.) & 1.00 (Ref.) \\
\hline Male & $0.71(0.41-1.22)$ & $0.90(0.60-1.33)$ & $0.30(0.19-0.46)^{b}$ & $0.57(0.29-1.06)$ & $1.29(0.93-1.79)$ & $1.02(0.74-1.40)$ \\
\hline \multicolumn{7}{|l|}{ Use public transportation } \\
\hline No & 1.00 (Ref.) & 1.00 (Ref.) & 1.00 (Ref.) & 1.00 (Ref.) & 1.00 (Ref.) & 1.00 (Ref.) \\
\hline Yes & $0.19(0.11-0.31)^{b}$ & $0.32(0.19-0.56)^{b}$ & $1.31(0.81-2.16)$ & $1.80(0.94-3.65)$ & $2.12(1.45-3.10)^{b}$ & $0.89(0.56-1.42)$ \\
\hline \multicolumn{7}{|l|}{ Living urban } \\
\hline No & 1.00 (Ref.) & 1.00 (Ref.) & 1.00 (Ref.) & 1.00 (Ref.) & 1.00 (Ref.) & 1.00 (Ref.) \\
\hline Yes & $0.87(0.45-1.61)$ & $1.37(0.92-2.03)$ & $1.70(1.03-2.90)^{d}$ & $0.68(0.35-1.27)$ & $1.52(1.09-2.11)^{d}$ & $1.59(1.15-2.20)^{c}$ \\
\hline \multicolumn{7}{|l|}{ Living alone } \\
\hline No & 1.00 (Ref.) & 1.00 (Ref.) & 1.00 (Ref.) & 1.00 (Ref.) & 1.00 (Ref.) & 1.00 (Ref.) \\
\hline Yes & $0.77(0.45-1.34)$ & $0.83(0.51-1.38)$ & $0.43(0.28-0.68)^{b}$ & $1.18(0.55-2.74)$ & $0.88(0.59-1.30)$ & $1.33(0.93-1.90)$ \\
\hline \multicolumn{7}{|l|}{ Income group } \\
\hline 1st quintile & 1.00 (Ref.) & 1.00 (Ref.) & 1.00 (Ref.) & 1.00 (Ref.) & 1.00 (Ref.) & 1.00 (Ref.) \\
\hline 2nd quintile & $1.11(0.52-2.38)$ & $1.31(0.69-2.52)$ & $1.00(0.53-1.87)$ & $2.21(0.9-5.99)$ & $1.17(0.69-1.98)$ & $1.6(1.02-2.52)^{d}$ \\
\hline 3rd quintile & $1.37(0.61-3.16)$ & $1.11(0.61-2.02)$ & $0.69(0.38-1.24)$ & $3.03(1.26-8.13)^{d}$ & $1.24(0.75-2.09)$ & $2.27(1.44-3.62)^{b}$ \\
\hline 4th quintile & $1.27(0.57-2.90)$ & $1.42(0.79-2.56)$ & $1.74(0.84-3.72)$ & $1.85(0.81-4.38)$ & $1.06(0.63-1.80)$ & $2.05(1.31-3.22)^{c}$ \\
\hline 5th quintile & $2.34(0.94-6.30)$ & $1.48(0.76-2.93)$ & $1.06(0.51-2.24)$ & $9.19(2.50-59.50)^{c}$ & $1.02(0.61-1.71)$ & $3.24(1.93-5.53)^{b}$ \\
\hline \multicolumn{7}{|l|}{ Feeling anxiety } \\
\hline No & 1.00 (Ref.) & 1.00 (Ref.) & 1.00 (Ref.) & 1.00 (Ref.) & 1.00 (Ref.) & 1.00 (Ref.) \\
\hline Yes & $1.40(0.81-2.46)$ & $0.9(0.60-1.34)$ & $2.11(1.37-3.31)^{b}$ & $1.67(0.89-3.24)$ & $1.04(0.76-1.43)$ & $1.31(0.96-1.80)$ \\
\hline Perceived susceptibility ${ }^{a}$ & $0.83(0.74-0.94)^{c}$ & $1.07(0.97-1.19)$ & $0.94(0.86-1.03)$ & $0.86(0.75-1.00)^{d}$ & $1.08(1.01-1.14)^{\mathrm{d}}$ & $1.00(0.94-1.07)$ \\
\hline Perceived severity ${ }^{a}$ & $0.91(0.83-0.99)^{d}$ & $1.05(0.97-1.13)$ & $1.00(0.92-1.10)$ & $0.97(0.87-1.10)$ & $1.05(0.99-1.12)$ & $1.07(1.02-1.14)^{d}$ \\
\hline \multicolumn{7}{|l|}{ Perceived effectiveness } \\
\hline Not effective at all & 1.00 (Ref.) & 1.00 (Ref.) & 1.00 (Ref.) & 1.00 (Ref.) & 1.00 (Ref.) & 1.00 (Ref.) \\
\hline Slightly effective & $1.11(0.04-32.35)$ & $1.51(0.63-3.74)$ & $2.16(1.01-4.62)^{d}$ & $2.66(0.52-14.81)$ & $1.92(1.03-3.68)^{d}$ & $1.66(0.85-3.27)$ \\
\hline Moderately effective & $0.23(0.01-1.51)$ & $1.09(0.54-2.17)$ & $2.59(1.28-5.19)^{c}$ & $2.65(0.65-10.52)$ & $4.08(2.35-7.40)^{b}$ & $2.45(1.32-4.58)^{c}$ \\
\hline Very effective & $0.50(0.02-3.16)$ & $3.79(1.83-7.72)^{b}$ & $3.64(1.69-7.87)^{b}$ & $7.99(1.96-31.42)^{c}$ & $6.03(3.35-11.31)^{b}$ & $3.03(1.65-5.56)^{b}$ \\
\hline Extremely effective & $0.51(0.03-3.14)$ & $4.14(2.08-8.02)^{b}$ & $3.59(1.75-7.30)^{b}$ & $7.89(1.91-31.63)^{c}$ & $9.23(5.14-17.31)^{b}$ & $4.81(2.61-8.92)^{b}$ \\
\hline
\end{tabular}

COVID-19, coronavirus diesease $2019 ; \mathrm{OR}$, odds ratio; $95 \% \mathrm{Cl}$, $95 \%$ confidence intervals

${ }^{\mathrm{a}}$ The odds ratios indicate indicate a propability of mask wearing with a $10 \%$ increase in each perception (\%)

${ }^{\text {b }} P$-value $<0.001$

${ }^{c} P$-value $<0.01$

${ }^{\mathrm{d}} P$-value $<0.05$

participants tended to engage in more preventive behaviors than did males. Consistent with previous studies on SARS [10, 11, 14, 21], we also found that older adults were more likely to take preventive measures. Highincome groups were associated with an increased probability of taking preventive behaviors, but educational experience and ethnicity may have been potential confounders underlying this association [22-24]. A study on sociodemographic factors in response to SARS in New
York suggested that high-income groups were more likely to access accurate information [25]. Therefore, high-income individuals may take more appropriate actions when faced with emerging diseases. We also assessed the effect of living environment on taking preventive behaviors. Given the high passenger density and difficulty of social distancing in public transportation, we would expect that those who use public transportation are more likely to take preventive measures. However, 
Table 4 Associations between public perceptions, personal characteristics, and handwashing/using hand sanitizers

\begin{tabular}{|c|c|c|c|c|c|c|}
\hline & $\begin{array}{l}\text { China } \\
\text { OR }(95 \% \mathrm{Cl})\end{array}$ & $\begin{array}{l}\text { Italy } \\
\text { OR }(95 \% \mathrm{Cl})\end{array}$ & $\begin{array}{l}\text { Japan } \\
\text { OR }(95 \% \mathrm{Cl})\end{array}$ & $\begin{array}{l}\text { Korea } \\
\text { OR }(95 \% \mathrm{Cl})\end{array}$ & $\begin{array}{l}\text { UK } \\
\text { OR }(95 \% \mathrm{Cl})\end{array}$ & $\begin{array}{l}\text { US } \\
\text { OR }(95 \% \mathrm{Cl})\end{array}$ \\
\hline \multicolumn{7}{|l|}{ Age group } \\
\hline Under 65 years old & 1.00 (Ref.) & 1.00 (Ref.) & 1.00 (Ref.) & 1.00 (Ref.) & 1.00 (Ref.) & 1.00 (Ref.) \\
\hline Over 65 years old & $1.75(0.71-5.11)$ & $1.74(0.56-7.64)$ & $2.47(1.27-5.21)^{d}$ & $0.55(0.26-1.28)$ & $3.50(0.66-64.78)$ & $2.20(0.70-9.77)$ \\
\hline \multicolumn{7}{|l|}{ Sex } \\
\hline Female & 1.00 (Ref.) & 1.00 (Ref.) & 1.00 (Ref.) & 1.00 (Ref.) & 1.00 (Ref.) & 1.00 (Ref.) \\
\hline Male & $0.56(0.31-0.98)^{d}$ & $0.55(0.23-1.23)$ & $0.32(0.19-0.53)^{b}$ & $0.52(0.26-1.00)$ & $0.43(0.16-1.06)$ & $0.47(0.21-1.01)$ \\
\hline \multicolumn{7}{|l|}{ Use public transportation } \\
\hline No & 1.00 (Ref.) & 1.00 (Ref.) & 1.00 (Ref.) & 1.00 (Ref.) & 1.00 (Ref.) & 1.00 (Ref.) \\
\hline Yes & $0.78(0.44-1.43)$ & $0.89(0.30-3.34)$ & $1.62(0.96-2.82)$ & $1.33(0.70-2.63)$ & $0.53(0.22-1.34)$ & $0.51(0.24-1.10)$ \\
\hline \multicolumn{7}{|l|}{ Living urban } \\
\hline No & 1.00 (Ref.) & 1.00 (Ref.) & 1.00 (Ref.) & 1.00 (Ref.) & 1.00 (Ref.) & 1.00 (Ref.) \\
\hline Yes & $1.29(0.66-2.43)$ & $1.31(0.57-2.96)$ & $0.66(0.40-1.10)$ & $0.51(0.25-1.00)$ & $0.57(0.23-1.39)$ & $1.01(0.48-2.09)$ \\
\hline \multicolumn{7}{|l|}{ Living alone } \\
\hline No & 1.00 (Ref.) & 1.00 (Ref.) & 1.00 (Ref.) & 1.00 (Ref.) & 1.00 (Ref.) & 1.00 (Ref.) \\
\hline Yes & $0.73(0.41-1.32)$ & $0.52(0.22-1.36)$ & $0.50(0.31-0.82)^{c}$ & $1.10(0.49-2.65)$ & $2.49(0.91-8.15)$ & $0.86(0.41-1.90)$ \\
\hline \multicolumn{7}{|l|}{ Income group } \\
\hline 1st quintile & 1.00 (Ref.) & 1.00 (Ref.) & 1.00 (Ref.) & 1.00 (Ref.) & 1.00 (Ref.) & 1.00 (Ref.) \\
\hline 2nd quintile & $0.51(0.21-1.15)$ & $0.52(0.14-1.81)$ & $1.02(0.53-1.96)$ & $2.71(1.00-8.69)$ & $1.00(0.27-3.61)$ & $2.35(0.74-8.33)$ \\
\hline 3rd quintile & $0.96(0.37-2.45)$ & $0.96(0.26-3.42)$ & $1.49(0.75-2.99)$ & $2.25(0.93-5.86)$ & $1.87(0.46-8.05)$ & $4.33(1.17-21.04)^{d}$ \\
\hline 4th quintile & $0.73(0.28-1.89)$ & $1.10(0.29-3.93)$ & $2.57(1.15-6.11)^{d}$ & $2.00(0.86-4.86)$ & $4.64(0.90-35.01)$ & $1.04(0.38-2.70)$ \\
\hline 5th quintile & $0.90(0.33-2.51)$ & $0.88(0.21-3.90)$ & $1.11(0.52-2.38)$ & $3.50(1.27-11.37)^{d}$ & $1.09(0.31-3.53)$ & $1.63(0.55-4.88)$ \\
\hline \multicolumn{7}{|l|}{ Feeling anxiety } \\
\hline No & 1.00 (Ref.) & 1.00 (Ref.) & 1.00 (Ref.) & 1.00 (Ref.) & 1.00 (Ref.) & 1.00 (Ref.) \\
\hline Yes & $2.05(1.15-3.80)^{d}$ & $1.36(0.59-3.35)$ & $1.74(1.09-2.83)^{d}$ & $1.16(0.61-2.22)$ & $0.84(0.35-2.07)$ & $1.06(0.52-2.23)$ \\
\hline Perceived susceptibility ${ }^{a}$ & $0.81(0.71-0.91)^{b}$ & $0.99(0.84-1.18)$ & $0.98(0.90-1.09)$ & $0.77(0.66-0.90)^{\mathrm{b}}$ & $1.05(0.89-1.27)$ & $0.91(0.80-1.03)$ \\
\hline Perceived severity ${ }^{a}$ & $1.02(0.93-1.13)$ & $1.05(0.90-1.25)$ & $0.96(0.88-1.06)$ & $1.12(0.98-1.30)$ & $1.01(0.85-1.21)$ & $0.92(0.81-1.06)$ \\
\hline \multicolumn{7}{|l|}{ Perceived effectiveness } \\
\hline Not effective at all & 1.00 (Ref.) & 1.00 (Ref.) & 1.00 (Ref.) & 1.00 (Ref.) & 1.00 (Ref.) & 1.00 (Ref.) \\
\hline Slightly effective & $0.40(0.02-2.92)$ & $1.85(0.41-7.86)$ & $3.00(0.97-9.33)$ & $4.55(0.65-35.65)$ & $1.01(0.18-4.64)$ & $1.45(0.28-6.80)$ \\
\hline Moderately effective & $0.38(0.02-2.08)$ & $6.84(1.51-28.73)^{c}$ & $4.53(1.53-13.43)^{c}$ & $2.10(0.40-10.27)$ & $3.37(0.62-15.17)$ & $4.26(0.82-20.61)$ \\
\hline Very effective & $0.79(0.04-4.26)$ & $16.15(3.52-68.93)^{b}$ & $7.75(2.60-23.09)^{b}$ & $8.28(1.57-41.10)^{c}$ & 3.35 (0.64-13.93) & $6.35(1.32-26.81)^{d}$ \\
\hline Extremely effective & $3.68(0.18-25.36)$ & $16.39(3.56-70.18)^{b}$ & $12.24(4.03-37.35)^{b}$ & $12.41(2.02-76.39)^{\complement}$ & $18.04(2.60-152.78)^{c}$ & $10.56(2.21-44.32)^{c}$ \\
\hline
\end{tabular}

COVID-19, coronavirus diesease $2019 ; \mathrm{OR}$, odds ratio; $95 \% \mathrm{Cl}$, $95 \%$ confidence intervals

${ }^{\mathrm{a}}$ The odds ratios indicate indicate a propability of handwashing with a $10 \%$ increase in each perception (\%)

${ }^{\mathrm{b}} P$-value $<0.001$

${ }^{\mathrm{c} P \text {-value }}<0.01$

${ }^{\mathrm{d}} P$-value $<0.05$

our results showed that the use of public transportation (as of the survey date) was negatively associated with the probability to wear a mask. This unexpected result may be explained by the downward bias of the coefficient estimate driven by the potential negative correlation between the covariate and unobserved variables. For instance, an individual who is more seriously concerned about a disease and thus more inclined to wear a mask may be more likely to avoid using public transportation.
To verify this potential endogeneity problem, we also ran a regression involving the use of public transportation before the pandemic, which may be correlated with the use of transportation after the pandemic will be independent of the unobserved variables. The results yielded theoretically consistent estimates, i.e., the use of public transportation was associated with a higher probability of wearing a mask. In addition, feeling anxiety may have been associated with preventive behaviors in 
Table 5 Associations between public perceptions, personal characteristics, and avoiding social gathering

\begin{tabular}{|c|c|c|c|c|c|c|}
\hline & $\begin{array}{l}\text { China } \\
\text { OR }(95 \% \mathrm{Cl})\end{array}$ & $\begin{array}{l}\text { Italy } \\
\text { OR }(95 \% \mathrm{Cl})\end{array}$ & $\begin{array}{l}\text { Japan } \\
\text { OR }(95 \% \mathrm{Cl})\end{array}$ & $\begin{array}{l}\text { Korea } \\
\text { OR }(95 \% \mathrm{Cl})\end{array}$ & $\begin{array}{l}\text { UK } \\
\text { OR }(95 \% \mathrm{Cl})\end{array}$ & $\begin{array}{l}\text { US } \\
\text { OR }(95 \% \mathrm{Cl})\end{array}$ \\
\hline \multicolumn{7}{|l|}{ Age group } \\
\hline Under 65 years old & 1.00 (Ref.) & 1.00 (Ref.) & 1.00 (Ref.) & 1.00 (Ref.) & 1.00 (Ref.) & 1.00 (Ref.) \\
\hline Over 65 years old & $2.78(1.29-6.55)^{d}$ & $1.89(0.84-4.88)$ & $1.15(0.57-2.49)$ & $1.22(0.65-2.41)$ & $1.28(0.60-3.01)$ & $1.38(0.80-2.47)$ \\
\hline \multicolumn{7}{|l|}{ Sex } \\
\hline Female & 1.00 (Ref.) & 1.00 (Ref.) & 1.00 (Ref.) & 1.00 (Ref.) & 1.00 (Ref.) & 1.00 (Ref.) \\
\hline Male & $0.81(0.52-1.26)$ & $0.82(0.49-1.37)$ & $0.33(0.19-0.58)^{b}$ & $0.93(0.60-1.42)$ & $0.91(0.57-1.45)$ & $0.54(0.36-0.82)^{c}$ \\
\hline \multicolumn{7}{|l|}{ Use public transportation } \\
\hline No & 1.00 (Ref.) & 1.00 (Ref.) & 1.00 (Ref.) & 1.00 (Ref.) & 1.00 (Ref.) & 1.00 (Ref.) \\
\hline Yes & $0.09(0.06-0.15)^{b}$ & $0.07(0.04-0.12)^{b}$ & $0.23(0.13-0.39)^{b}$ & $0.15(0.10-0.23)^{b}$ & $0.07(0.04-0.11)^{b}$ & $0.07(0.04-0.11)^{b}$ \\
\hline \multicolumn{7}{|l|}{ Living urban } \\
\hline No & 1.00 (Ref.) & 1.00 (Ref.) & 1.00 (Ref.) & 1.00 (Ref.) & 1.00 (Ref.) & 1.00 (Ref.) \\
\hline Yes & $1.64(0.99-2.70)$ & $0.43(0.24-0.75)^{c}$ & $0.88(0.51-1.55)$ & $2.24(1.45-3.46)^{b}$ & $0.49(0.31-0.78)^{c}$ & $1.45(0.96-2.24)$ \\
\hline \multicolumn{7}{|l|}{ Living alone } \\
\hline No & 1.00 (Ref.) & 1.00 (Ref.) & 1.00 (Ref.) & 1.00 (Ref.) & 1.00 (Ref.) & 1.00 (Ref.) \\
\hline Yes & $0.84(0.53-1.34)$ & $0.51(0.29-0.94)^{d}$ & $1.12(0.61-2.16)$ & $1.10(0.62-2.04)$ & $1.75(1.01-3.11)$ & $0.82(0.51-1.32)$ \\
\hline \multicolumn{7}{|l|}{ Income group } \\
\hline 1st quintile & 1.00 (Ref.) & 1.00 (Ref.) & 1.00 (Ref.) & 1.00 (Ref.) & 1.00 (Ref.) & 1.00 (Ref.) \\
\hline 2nd quintile & $0.95(0.48-1.89)$ & $0.64(0.27-1.50)$ & $2.02(0.81-5.06)$ & $0.90(0.46-1.76)$ & $0.75(0.35-1.59)$ & $0.68(0.34-1.36)$ \\
\hline 3rd quintile & $0.53(0.27-1.05)$ & $0.71(0.31-1.58)$ & $1.12(0.48-2.56)$ & $0.65(0.35-1.19)$ & $0.76(0.36-1.58)$ & $0.81(0.41-1.61)$ \\
\hline 4th quintile & $0.96(0.46-2.01)$ & $0.75(0.33-1.67)$ & $0.94(0.40-2.14)$ & $1.08(0.55-2.14)$ & $0.81(0.37-1.77)$ & $0.64(0.33-1.23)$ \\
\hline 5th quintile & $1.10(0.52-2.36)$ & $0.95(0.38-2.43)$ & $1.15(0.45-2.93)$ & $0.80(0.40-1.60)$ & $0.73(0.34-1.51)$ & $0.49(0.24-0.97)^{d}$ \\
\hline \multicolumn{7}{|l|}{ Feeling anxiety } \\
\hline No & 1.00 (Ref.) & 1.00 (Ref.) & 1.00 (Ref.) & 1.00 (Ref.) & 1.00 (Ref.) & 1.00 (Ref.) \\
\hline Yes & $1.10(0.70-1.73)$ & $0.63(0.37-1.06)$ & $1.10(0.64-1.87)$ & $1.10(0.73-1.67)$ & $1.19(0.75-1.91)$ & $1.12(0.75-1.70)$ \\
\hline Perceived susceptibility ${ }^{a}$ & $0.74(0.66-0.83)^{b}$ & $0.96(0.86-1.07)$ & $0.85(0.77-0.95)^{c}$ & $0.81(0.73-0.91)^{\mathrm{b}}$ & $0.97(0.89-1.06)$ & $0.83(0.77-0.90)^{\mathrm{b}}$ \\
\hline Perceived severity ${ }^{a}$ & $1.27(1.16-1.41)^{\mathrm{b}}$ & $0.98(0.89-1.09)$ & $0.97(0.87-1.08)$ & $1.05(0.97-1.14)$ & $0.92(0.84-1.00)$ & $1.11(1.03-1.19)^{c}$ \\
\hline \multicolumn{7}{|l|}{ Perceived effectiveness } \\
\hline Not effective at all & 1.00 (Ref.) & 1.00 (Ref.) & 1.00 (Ref.) & 1.00 (Ref.) & 1.00 (Ref.) & 1.00 (Ref.) \\
\hline Slightly effective & $0.73(0.20-2.66)$ & $0.70(0.17-2.68)$ & $0.42(0.10-1.53)$ & $1.39(0.33-5.64)$ & $1.46(0.42-5.05)$ & $2.25(0.81-6.27)$ \\
\hline Moderately effective & $1.09(0.36-3.02)$ & $0.95(0.26-3.25)$ & $0.41(0.11-1.21)$ & $2.53(0.68-9.10)$ & $0.85(0.30-2.27)$ & $3.86(1.48-9.97)^{c}$ \\
\hline Very effective & $2.39(0.79-6.56)$ & $4.48(1.25-14.99)^{d}$ & $0.86(0.22-2.74)$ & $3.38(0.97-11.21)^{d}$ & $2.08(0.76-5.52)$ & $4.16(1.70-10.00)^{c}$ \\
\hline Extremely effective & $3.79(1.28-10.23)^{d}$ & $3.09(0.94-9.09)$ & $1.28(0.34-3.82)$ & $6.18(1.77-20.60)^{c}$ & $4.45(1.63-11.63)^{c}$ & $4.34(1.84-9.95)^{b}$ \\
\hline
\end{tabular}

COVID-19, coronavirus diesease 2019; OR, odds ratio; $95 \% \mathrm{Cl}$, 95\% confidence intervals

${ }^{\text {a }}$ The odds ratios indicate indicate a propability of avoiding gathering with a $10 \%$ increase in each perception (\%)

${ }^{\mathrm{b}} P$-value $<0.001$

${ }^{\mathrm{c} P \text {-value }}<0.01$

${ }^{\mathrm{d} P \text {-value }}<0.05$

some countries, which was consistent with the results observed in previous studies [21, 25].

Behavioral changes triggered by conformity and recommendations from others showed different impacts on preventive behaviors across countries, and the impacts also varied across all three preventive behaviors within each country. However, the bottom line is that individuals who changed their behavior because of recommendations from doctors/public health officials were more likely to take preventive behaviors in many countries, e.g., wearing a mask in China, Italy, Japan, and the USA, and avoiding social gatherings in the USA. On the other hand, recommendations by politicians did not significantly affect preventive behaviors, except for wearing a mask in the USA and avoiding social gatherings in the UK. In many countries during the pandemic, local 
Table 6 Associations between conformity and recommendations from others and three preventive behaviors

\begin{tabular}{|c|c|c|c|c|c|c|}
\hline & China & Italy & Japan & Korea & UK & US \\
\hline & OR $(95 \% \mathrm{Cl})$ & OR $(95 \% \mathrm{Cl})$ & OR $(95 \% \mathrm{Cl})$ & OR $(95 \% \mathrm{Cl})$ & OR $(95 \% \mathrm{Cl})$ & OR $(95 \% \mathrm{Cl})$ \\
\hline \multicolumn{7}{|l|}{ Wearing a mask } \\
\hline Conformity & $2.36(0.74-10.74)$ & $1.13(0.45-3.49)$ & $5.18(1.54-32.35)^{c}$ & $2.62(0.83-12.08)$ & $0.78(0.49-1.21)$ & $1.29(0.83-2.05)$ \\
\hline $\begin{array}{l}\text { Recommendations from } \\
\text { family/friends }\end{array}$ & $1.33(0.70-2.70)$ & $0.68(0.33-1.53)$ & $3.42(1.19-14.48)^{c}$ & $1.05(0.42-3.10)$ & $1.41(0.90-2.19)$ & $1.27(0.88-1.84)$ \\
\hline $\begin{array}{l}\text { Recommendations from } \\
\text { doctors/public health officials }\end{array}$ & $2.04(1.12-3.78)^{c}$ & $2.02(1.34-3.06)^{\mathrm{a}}$ & $2.84(1.34-7.01)^{c}$ & $1.24(0.51-3.53)$ & $0.66(0.48-0.90)^{b}$ & $1.82(1.33-2.50)^{\mathrm{a}}$ \\
\hline $\begin{array}{l}\text { Recommendations from } \\
\text { politicians }\end{array}$ & $1.00(0.47-2.31)$ & $1.60(0.98-2.72)$ & $1.39(0.67-3.30)$ & $1.09(0.26-8.33)$ & $0.56(0.41-0.77)^{a}$ & $1.63(1.15-2.33)^{b}$ \\
\hline \multicolumn{7}{|l|}{ Handwashing/using hand sanitizers } \\
\hline Conformity & $0.59(0.23-1.73)$ & $0.94(0.17-17.83)$ & $1.22(0.52-3.39)$ & $1.43(0.51-5.22)$ & $0.44(0.18-1.21)$ & $0.70(0.30-1.78)$ \\
\hline $\begin{array}{l}\text { Recommendations from } \\
\text { family/friends }\end{array}$ & $0.96(0.51-1.89)$ & $0.60(0.15-3.99)$ & $1.52(0.62-4.61)$ & $1.71(0.60-6.32)$ & $1.44(0.39-9.40)$ & $0.90(0.41-2.15)$ \\
\hline $\begin{array}{l}\text { Recommendations from } \\
\text { doctors/public health officials }\end{array}$ & $1.70(0.92-3.20)$ & $2.42(0.98-6.34)$ & $1.75(0.85-4.13)$ & $2.85(0.95-12.42)$ & $0.97(0.40-2.37)$ & $1.71(0.81-3.79)$ \\
\hline $\begin{array}{l}\text { Recommendations from } \\
\text { politicians }\end{array}$ & $1.74(0.74-4.88)$ & $0.60(0.24-1.63)$ & $1.21(0.55-3.05)$ & $1.83(0.32-36.37)$ & $0.91(0.38-2.24)$ & $2.68(0.99-9.42)$ \\
\hline \multicolumn{7}{|l|}{ Avoiding social gathering } \\
\hline Conformity & $0.78(0.34-1.93)$ & $0.76(0.26-2.67)$ & $1.41(0.54-1.51)$ & $1.11(0.58-2.27)$ & $0.85(0.45-1.66)$ & $1.20(0.70-2.15)$ \\
\hline $\begin{array}{l}\text { Recommendations from } \\
\text { family/friends }\end{array}$ & $1.17(0.68-2.05)$ & $0.48(0.21-1.24)$ & $1.13(0.46-3.25)$ & $0.73(0.38-1.46)$ & $0.72(0.38-1.41)$ & $1.23(0.76-2.04)$ \\
\hline $\begin{array}{l}\text { Recommendations from } \\
\text { doctors/public health officials }\end{array}$ & $1.22(0.75-1.98)$ & $1.54(0.90-2.64)$ & $1.44(0.64-3.70)$ & $1.40(0.77-2.68)$ & $1.05(0.66-1.68)$ & $2.14(1.40-3.28)^{\mathrm{a}}$ \\
\hline $\begin{array}{l}\text { Recommendations from } \\
\text { politicians }\end{array}$ & $0.66(0.36-1.22)$ & $1.16(0.62-2.28)$ & $1.09(0.46-2.93)$ & $0.94(0.33-3.46)$ & $1.70(1.05-2.79)^{b}$ & $1.44(0.90-2.34)$ \\
\hline
\end{tabular}

Adjusted for gender, age (65 or more), residential area, living arrangement, income levels (five groups), perceived probability, perceived severity, perceived effectiveness, feeling anxiety, and region

COVID-19, coronavirus diesease 2019; OR, odds ratio; $95 \% \mathrm{Cl}, 95 \%$ confidence intervals

${ }^{\text {a }} P$-value $<0.001$

${ }^{\mathrm{b}} P$-value $<0.01$

${ }^{c} P$-value $<0.05$

governments have been holding regular press conferences to provide daily updates on the pandemic and call for preventive measures. Our results suggest that the engagement of medical professionals in addressing the significance of preventive measures is more effective. Another interesting finding was that individuals in Japan who changed their behavior after taking conformity into account were more likely to wear a mask. This may reflect cultural norms in Japan, in which individual behavior is affected greatly by the behaviors of others around him/her [26, 27].

Several methodological issues need to be mentioned as limitations. First, this study was conducted in a limited sample population, which may affect the external and internal validity. Ideally, an epidemiological study should be conducted on randomly selected participants from the general population. This online survey was designed to retain national representation regarding basic demographic variables, which would alleviate a fundamental sampling bias. However, detailed information on response rate, recruiting method, and the amount of remuneration were not available, which constitute potential sources of bias. Despite this, we consider that this sampling method should be accepted given the nature of an emergency survey. Second, important covariates such as ethnicity, educational experience, and preexisting comorbidities were not available in this study. Previous studies have reported that ethnicity is a major factor in preventive behaviors [23]. Third, the design of questionnaire used in this study may be crucial for interpreting the results. In particular, the question item on perceived susceptibility to COVID-19 was likely critically problematic. In this survey, participants were asked "What do you think is the probability that you are or have been infected with COVID-19?," i.e., the current susceptibility for COVID-19. However, in general, a question on perceived susceptibility should ask about the possibility of being infected in the future. In addition, the questionnaire had only one question regarding personal perceptions, whereas conventional methods usually employ 
multiple questions to assess individual perceptions more precisely. Due to the poorly defined questionnaire and the cross-sectional design of this study, some caution is needed in interpreting the results, as described above. Further cross-country studies using a longitudinal design and well-structured questionnaires could be expected to overcome these limitations and provide a more accurate assessment of the structural relationships between different factors and preventive behaviors. Forth, we need to be more careful when interpreting the results, which showed wide confidence intervals. In particular, since almost all people practiced handwashing in each country, the confidence intervals for the estimates were wider than the other two preventive behaviors. We may potentially reduce the number of variables in the regressions. However, given the emphasis on comparing the results across different preventive practices, we used the same set of variables for each preventive behavior rather than making variable selection. Therefore, for a wide confidence interval, the estimates should be interpreted by integrating them with other studies. Fifth, in comparing the results among six different counties, we would need to take into account the different epidemic phases of COVID-19 in each country at the time of the survey. In the fully adjusted models, we included regional fixed effects to control for the heterogeneous infectious status within a country. However, given that the population are firstly stratified by country, it was not straightforward to incorporate the across-country heterogeneity. It is important to keep this issue in mind when interpreting the data. The infection status at the time of the online survey has been described in previous studies [19].

\section{Conclusions}

In conclusion, first, our results suggest that, when encouraging the general public to engage in preventive measures during a pandemic, it would be effective to publicize the effectiveness of such measures. Second, associations between individual characteristics (both demographic and socioeconomic) and preventive behaviors vary across countries and preventive measures, which highlights the importance of targeting subgroups of people when preventive measures are implemented by a health administration. Third, our results suggest that incorporating different associations between individual characteristics and preventive behaviors across countries may provide more precise simulation results in mathematical modeling.

\section{Abbreviations}

Cl: Confidence interval; COVID-19: Coronavirus disease 2019; HBM: Health Belief Model; OR: Odds ratio; SARS: Severe acute respiratory syndrome; SARSCoV-2: Severe acute respiratory syndrome-coronavirus-2

\section{Supplementary Information}

The online version contains supplementary material available at https://doi. org/10.1186/s12199-021-00952-2.

Additional file 1. The online self-administered questionnaire for this sixcountry survey. This questionnaire was used to assess individual perceptions of COVID-19, and their behavioral change.

\section{Acknowledgements}

We have a tremendous amount of respect and gratitude for the following researchers who designed and executed the online survey and made information available to the public: Dr. Michele Belot, Dr. Syngjoo Choi, Dr. Julian C. Jamison, Dr. Nicholas W. Papageorge, Dr. Egon Tripodi, and Dr. Eline Van den Broek-Altenburg. We are also grateful to Dr. Natsuki Kondo (Nagoya University) and Dr. Megumi Fujii (Japanese Red Cross Toyota College of Nursing) for their comments on the initial research ideas.

\section{Authors' contributions}

R.F. and K.S. wrote the manuscript; R.F., K.S., and J.N. performed statistical analysis; K.S. corrected data from online sources; R.F. and K.S. were in charge of data management; J.N. contributed to the revision of the manuscript critically for important intellectual content. All the authors reviewed the manuscript. The author(s) read and approved the final manuscript.

Availability of data and materials

The data are publicly available at https://osf.io/aubkc/. The codes used for the statistical analysis are uploaded to GitHub (https://github.com/ fujichaaan/covid19_opendata)

\section{Declarations}

Ethics approval and consent to participate

The original study protocol was approved by the ethics board at the University of Exeter (eUEBS003014 v2.0). In addition, the protocol was officially reviewed by the ethics review board of Fujita Health University (HM20-182). Written informed consent was obtained from all participants through an online form.

\section{Consent for publication}

This study used publicly available data. Before participating in the online survey, informed consent was obtained from all participants after specifying that anonymized individual-level data would be made public.

\section{Competing interests}

The authors declare that they have no competing interests.

\section{Author details}

${ }^{1}$ Department of Preventive Medical Sciences, Fujita Health University School of Medical Sciences, 1-98 Dengakugakubo, Kutsukake-cho, Toyoake 470-1192, Japan. ${ }^{2}$ Department of Economics, The Pennsylvania State University, University Park, PA 16802, USA. ${ }^{3}$ Department of Business Management, Meijo University, 1-501 Shiogamaguchi, Tempaku-ku, Nagoya 468-8502, Japan.

Received: 14 January 2021 Accepted: 22 February 2021

Published online: 03 March 2021

\section{References}

1. World Health Organization. Coronavirus disease 2019 (COVID-19) situation report - 207. Accessed at https://www.who.int/docs/default-source/corona viruse/situation-reports/20200814-covid-19-sitrep-207.pdf?sfvrsn=2f2154e6_2 on 14 August 2020.

2. Worldometer. COVID-19 Coronavirus outbreak. Accessed at https://www. worldometers.info/coronavirus/ on 14 January 2021.

3. CDC COVID-19 Response Team. Severe outcomes among patients with coronavirus disease 2019 (COVID-19) - United States, February 12-March 16, 2020. MMWR Morb Mortal Wkly Rep. 2020;69:343-6.

4. CDC COVID-19 Response Team. Preliminary estimates of the prevalence of selected underlying health conditions among patients with coronavirus disease 2019 - United States, February 12-March 28, 2020. MMWR Morb Mortal Wkly Rep. 2020;69:382-6. 
5. Pan A, Liu L, Wang C, Guo H, Hao X, Wang Q, et al. Association of Public health interventions with the epidemiology of the COVID-19 outbreak in Wuhan, China. JAMA. 2020;323:1-9.

6. Davies NG, Kucharski AJ, Eggo RM, Gimma A, Edmunds WJ. Centre for the Mathematical Modelling of Infectious Diseases COVID-19 working group. Effects of non-pharmaceutical interventions on COVID-19 cases, deaths, and demand for hospital services in the UK: a modelling study. Lancet Public Health. 2020;5:e375-85.

7. Chu DK, Akl EA, Duda S, Solo K, Yaacoub S, Schünemann HJ. COVID-19 Systematic Urgent Review Group Effort (SURGE) study authors. COVID-19 Systematic Urgent Review Group Effort (SURGE) study authors. Physical distancing, face masks, and eye protection to prevent person-to-person transmission of SARS-CoV-2 and COVID-19: a systematic review and metaanalysis. Lancet. 2020;395:1973-87.

8. Cowling BJ, Ali ST, Ng TWY, Tsang TK, Li JCM, Fong MW, et al. Impact assessment of non-pharmaceutical interventions against coronavirus disease 2019 and influenza in Hong Kong: an observational study. Lancet Public Health. 2020;5:e279-8.

9. Feng S, Shen C, Xia N, Song W, Fan M, Cowling BJ. Rational use of face masks in the COVID-19 pandemic. Lancet Respir Med. 2020;8:434-6.

10. Tang CS, Wong CY. Factors influencing the wearing of facemasks to prevent the severe acute respiratory syndrome among Chinese in Hong Kong. Prev Med. 2004;39:1193.

11. Tang CS, Wong CY. An outbreak of the severe acute respiratory syndrome: predictors of health behaviors and effect of community prevention measures in Hong Kong, China. Am J Public Health. 2003;93: 1887-8.

12. Leung GM, Quah S, Ho LM, Ho SY, Hedley AJ, Lee HP, et al. A tale of two cities: community psychobehavioral surveillance and related impact on outbreak control in Hong Kong and Singapore during the severe acute respiratory syndrome epidemic. Infect Control Hosp Epidemiol. 2004;25: 1033-41.

13. Bruine de Bruin W, Bennett D. Relationships between initial COVID-19 risk perceptions and protective health behaviors: a national survey. Am J Prev Med. 2020;59:157-67.

14. Lau JTF, Yang $\mathrm{X}$, Tsui $\mathrm{H}$, Kim JH. Monitoring community responses to the SARS epidemic in Hong Kong: from day 10 to day 62. J Epidemiol Community Health. 2003;57:864-70.

15. Pampel FC, Krueger PM, Denney JT. Socioeconomic disparities in health behaviors. Annu Rev Sociol. 2010;36:349-70. https://doi.org/10.1146/a nnurev.soc.012809.102529.

16. Bertozzi AL, Franco E, Mohler G, Short MB, Sledge D. The challenges of modeling and forecasting the spread of COVID-19. Proc Natl Acad Sci USA. 2020;117:16732-8.

17. Kissler SM, Tedijanto C, Goldstein E, Grad YH, Lipsitch M. Projecting the transmission dynamics of SARS-CoV-2 through the postpandemic period. Science. 2020;368:860-8.

18. Giordano G, Blanchini F, Bruno R, Colaneri P, Di Filippo A, Di Matteo A, et al. Modelling the COVID-19 epidemic and implementation of population-wide interventions in Italy. Nat Med. 2020;26:855-60.

19. Belot M, Choi S, Jamison JC, Papageorge NW, Tripodi E, van den BroekAltenburg E. Six-country survey on COVID-19. Work Paper Database. 2020; https://osf.io/aubkc/.

20. Clark N, Becker M. Theoretical models and strategies for improving adherence and disease management. In: Shumaker S, Schron E, Ockene J, McBee W, editors. The handbook of health behavior change. 2nd ed. New York: Springer Publishing Company; 1998. p. 5-32

21. Leung GM, Ho LM, Chan SK, Ho SY, Bacon-Shone J, Choy RY, et al. Longitudinal assessment of community psychobehavioral responses during and after the 2003 outbreak of severe acute respiratory syndrome in Hong Kong. Clin Infect Dis. 2005;40:1713-20.

22. Laaksonen $M$, Prättälä $R$, Helasoja V, Uutela A, Lahelma E. Income and health behaviours. Evidence from monitoring surveys among Finnish adults. J Epidemiol Community Health. 2003:57:711-7.

23. Rubin GJ, Amlôt R, Page L, Wessely S. Public perceptions, anxiety, and behaviour change in relation to the swine flu outbreak: cross sectional telephone survey. BMJ. 2009;339:b2651.

24. Wolf MS, Serper M, Opsasnick L, O'Conor RM, Curtis L, Benavente JY, et al. Awareness, attitudes, and actions related to COVID-19 among adults with chronic conditions at the onset of the U.S. outbreak: a cross-sectional survey. Ann Intern Med. 2020;173:100-9.
25. Des Jarlais DC, Galea S, Tracy M, Tross S, Vlahov D. Stigmatization of newly emerging infectious diseases: AIDS and SARS. Am J Public Health. 2006;96:561-7.

26. Cialdini RB, Goldstein NJ. Social influence: compliance and conformity. Annu Rev Psychol. 2004;55:591-621.

27. Abrams D, Wetherell M, Cochrane S, Hogg MA, Turner JC. Knowing what to think by knowing who you are: self-categorization and the nature of norm formation, conformity and group polarization. $\mathrm{Br} J \mathrm{Soc}$ Psychol. 1990;29:97-119.

\section{Publisher's Note}

Springer Nature remains neutral with regard to jurisdictional claims in published maps and institutional affiliations.
Ready to submit your research? Choose BMC and benefit from:

- fast, convenient online submission

- thorough peer review by experienced researchers in your field

- rapid publication on acceptance

- support for research data, including large and complex data types

- gold Open Access which fosters wider collaboration and increased citations

- maximum visibility for your research: over $100 \mathrm{M}$ website views per year

At $\mathrm{BMC}$, research is always in progress.

Learn more biomedcentral.com/submissions 